\title{
ASPECTOS DA ALIMENTAC̄̃̃O DE TANYSTYLUM ISABELLAE MARCUS E ANOPLODACTYLUS STICTUS MARCUS (PANTOPODA)
}

\author{
Franci Mary Fantinato Varoli ${ }^{1}$
}

\begin{abstract}
FEEDING ASPECTS OF TANYSTYLUM ISABELLAE AND ANOPLODACTYLUS STICTUS (PANTOPODA). This work deals with feeding tess performed in laboratory with Pantopoda species, Tanystylum isabellae Marcus, 1940 and Anoplodactylus stictus Marcus, 1940. Animals (alive and dead) and vegetable foods were offered to males without eggs, males with eggs, females and juveniles Pantopoda. Of the 13 types of food offered to the animals only Sertularia Linnaeus, 1758 (Hydrozoa), Amathia distans Busk, 1886 (Bryozoa), Hyale media (Dana, 1853) (Crustacea, Gammaridea), Caprella danilevskii Czerniavski, 1868 (Crustacea-Caprellidae), Artemia salina Leach, 1812 (Crustacea, Anostraca) e Perna perna (Linné, 1758) (Bivalvia, Mytilidae) were actually eaten. The macroalgae Sargassum C. Agardh, 1820; Padina Adanson, 1763; Chaetomorpha Kützing, 1845 and Ulva Linnaeus, 1753, the Hydrozoa Dynamena (Linnaeus, 1758), Polychaeta and anemon fragments weren't accepted.
\end{abstract}

KEYWORDS. Pantopoda, Sargassum fauna, feeding

Pantópodes são integrantes da fauna do fital, mas a sua ecologia não é muito conhecida. No fital de Ubatuba e Itanhaém, Tanystylum isabellae Marcus, 1940 e Anoplodactylus stictus Marcus, 1940 são usualmente as espécies mais abundantes no Sargassum sp. (VAROLi 1981).

Ainda que vários estudos sobre pantópodes tenham sido realizados sob diferentes aspectos em diversas localidades, trabalhos sobre seus hábitos alimentares constituem a minoria (STOCK 1978; ARNAUD 1978; SLOAN 1979; BAMBER \& DAVIS 1982; CHILD \& HARBISON 1986).

O objetivo deste estudo foi avaliar a ingestão ou não de determinados alimentos por jovens, fềmeas, machos sem ovos e machos ovados de Tanystylum isabellae e Anoplodactylus stictus em laboratório.

\section{MATERIAL E MÉTODOS}

Tufos de Sargassum C. Agardh, 1820 foram retirados do substrato em maio de 1978, em Itanhaém (São Paulo) e colocados em cuba com água do mar. Os pantópodes encontrados foram transferidos para uma cuba menor, também com água do mar, e separados por espécie. No laboratório, manteve-se os indivíduos coletados (28) em béqueres com água do mar, em condições mais próximas possíveis de temperatura $\left(25^{\circ} \mathrm{C}\right)$, salinidade $(33)$ e oxigênio $(4,5 \mathrm{ml} / \mathrm{l})$ a que foram

1) Instituto Oceanográfico, Universidade de São Paulo, Caixa Postal 9075, 01065-970 São Paulo, São Paulo, Brasil. Pesquisadora do CNPq. 
encontrados, permanecendo em jejum por 24 horas. Ao término desse período, para cada indivíduo foi dada uma categoria de alimento, realizando-se observações após o transcurso de 24 horas. Procedeu-se da seguinte maneira: se o indivíduo tivesse ingerido alimento, ficaria outras 24 horas em novo jejum para o possível esvaziamento do trato digestivo; caso contrário, novo alimento era oferecido.

Treze tipos de alimento foram oferecidos para 18 espécimens de Tanystylum isabellae (quatro machos sem ovos, dois machos ovados, sete fêmeas e cinco jovens) e 10 indivíduos de Anoplodactylus stictus (dois machos sem ovos, cinco fêmeas e três jovens).

Os animais utilizados como alimento foram conservados vivos em um aquário devidamente aerado. Sertularia Linnaeus, 1758, Dynamena (Linnaeus, 1758) (Hydrozoa) e Amathia distans Busk, 1886 (Bryozoa) foram oferecidos vivos, contados os hidrantes e zoóides, respectivamente, e observados antes e depois, para se comprovar a provável ingestão. Actínia, Perna perna (Linné, 1758) (Mytilidae) e Polychaeta e, as algas, Sargassum C. Agardh, 1820, Padina Adanson, 1763, Chaetomorpha Kützing, 1845 e Ulva Linnaeus, 1753 foram cortados em pequenos pedaços, observados ao microscópio e desenhados antes e depois, com o propósito de se constatar a ingestão ou não do alimento. Hyale media (Dana, 1853) (Gammaridea), Cuprella danilevskii Czerniavski, 1868 (Caprellidae) e Artemia salina Leach, 1812 (Anostraca) foram dados mortos (partes) e vivos.

Ao término do experimento foi feita uma análise descritiva, que consta na tabela I.

\section{RESULTADOS}

Dos 13 tipos de alimentos oferecidos Sertularia sp. foi consumido pelas duas espécies estudadas. Observou-se que os hidrantes vivos oferecidos após 24 horas estavam vazios, enquanto que Dynamena sp. não foi ingerido. Quanto a Amathia distans, as observações restringiram-se ao trato digestivo dos pantópodes pelas dificuldades em diferenciar-se o briozoário com conteúdo ou não, porém, admite-se sua ingestão pois observou-se que os intestinos dos pantópodes permaneciam cheios. Já as porções de actínias oferecidas não foram ingeridas. Os crustáceos, Hyale media, Caprella danilevskii e Artemia salina e o bivalve Perna perna foram comidos pelos pantópodes, porém, partes do animal. As porções de poliquetos oferecidas foram rejeitadas e os crustáceos quando colocados vivos não eram predados. As algas oferecidas ( Sargassum, Padina, Chaetomorpha e Ulva) foram rejeitadas.

Pouco se pode afirmar quanto a diferenças alimentares entre jovens, fêmeas, machos sem ovos e machos ovados. Foi entretanto constatado, através das porções de alimentos oferecidas, que machos ovados comeram Sertularia sp. e Hyale media em menor quantidade que os demais indivíduos. Em relação aos jovens, pode-se verificar que eles se alimentaram das mesmas quantidades de alimentos que machos e fêmeas. 
Tabela I. Alimentos testados para as duas espécies de Pantopoda comos correspondentes dias de duração de cada grupo de indivíduo.

\begin{tabular}{|c|c|c|c|c|}
\hline Espécie & $\begin{array}{l}\text { Número } \\
\text { individuos }\end{array}$ & $\begin{array}{l}\text { Duração em } \\
\text { Laboratório (dias) }\end{array}$ & Alimento ingerido & Alımento não ingerido \\
\hline \multirow[t]{4}{*}{ T. isabellae } & $\begin{array}{l}2 \text { machos } \\
\text { com ovos }\end{array}$ & $2-9$ & Sertularia sp.; Hyale medica (morto) & $\begin{array}{l}\text { Hyale media (vivo); Polychaeta; } \\
\text { Sargassum; Chaetomorpha }\end{array}$ \\
\hline & $\begin{array}{l}4 \text { machos } \\
\text { sem ovos }\end{array}$ & $2 \cdot 56$ & $\begin{array}{l}\text { Sertularia sp.; Amathia distans; } \\
\text { Hyale media (morto); Artemia } \\
\text { salina (morto) }\end{array}$ & $\begin{array}{l}\text { Dynamena sp. actínia; Caprella } \\
\text { danilevskii (vivo): Padina; } \\
\text { Sargassum; Chaetomompha; Ulva; } \\
\text { Artemia salina (vivo) }\end{array}$ \\
\hline & 7 têmeas & $4-56$ & $\begin{array}{l}\text { Sertularia sp.; Amathia distans; } \\
\text { Artemia salina (mortol; Perna perna }\end{array}$ & $\begin{array}{l}\text { Dynamena sp.; actinia; Hyale } \\
\text { media (vivo); Artemia salina (vivol; } \\
\text { Ulva }\end{array}$ \\
\hline & 5 jovens & $4-56$ & $\begin{array}{l}\text { Sertularia sp.; Amathia distans; } \\
\text { Caprella danilevskil !mortol; Perna } \\
\text { perna }\end{array}$ & $\begin{array}{l}\text { Dynamena sp.; actinia; Polychaeta; } \\
\text { Sargassum; Ulva }\end{array}$ \\
\hline \multirow[t]{3}{*}{ A. stictus } & $\begin{array}{l}2 \text { machos } \\
\text { sem ovos }\end{array}$ & $37-43$ & $\begin{array}{l}\text { Sertularia sp.; Amathia distans; } \\
\text { Perna perna }\end{array}$ & Dynamena sp.; actinia; U/va \\
\hline & 5 fêmeas & $4 \cdot 47$ & $\begin{array}{l}\text { Sertularia sp.: Amathia distans; } \\
\text { Hyale media (morto); Caprella } \\
\text { danilevskii (morto): Artemia salina } \\
\text { (morto) }\end{array}$ & $\begin{array}{l}\text { Dynamena sp.; actinia; Hyale } \\
\text { media (vivol; Caprella danilevskii } \\
\text { (vivol; Artemia salina (vivo); } \\
\text { Polychaeta; Padina; Sargassum; } \\
\text { Chaetomorpha; Ulva }\end{array}$ \\
\hline & 3 jovens & $58-60$ & Sertularia sp.; Amathia distans & $\begin{array}{l}\text { Dynamena sp.; actínia; Sargassum; } \\
\text { Ulva }\end{array}$ \\
\hline
\end{tabular}

\section{DISCUSSÃO E CONCLUSÕES}

Constatou-se a ingestão de Sertularia sp. por ambas espécies de pantópodes, o que está de acordo com DE HARO (1967). STOCK (1978) testou Dynamena pumila Lamouroux, 1812 para três diferentes espécies de pantópodes e observou que apenas uma delas o consumia, o que, no entanto, não ocorreu para nenhuma das espécies testadas neste experimento.

Apesar de actínia ser muito comum no local onde os pantópodes foram coletados, foi um dos alimentos rejeitados. FRY (1965), no entanto, observou alguns indivíduos de Pycnogonum stérnsi Ives, 1892 com suas probóscides inseridas ou próximas a anêmonas Anthopleura xanthogrammica Brandt, 1835 e Bunodactis elegantissima (Brandt, 1835). Outros autores como STOCK (1978) e CHILD \& HARBISON (1986) descreveram pantópodes alimentando-se de actínias ou cifomedusas vivas, porém, o fato dos resultados aqui obtidos serem contrários aos dos autores mencionados é provavelmente devido ao muco emitido pelos pedaços das actínias mortas.

A ingestão do briozoário Amathia distans também é fato conhecido. Segundo dados da literatura, hidrozoários e briozoários fazem parte da dieta dos pantópodes por serem facilmente predados por eles.

Os dados obtidos neste experimento revelam que Tanystylum isabellae e Anoplodactylus stictus comem bivalves. Esses resultados já haviam sido obtidos por autores como ANDRÉ \& LAMY (1938) e OSHIMA (1927; 1933 a,b).

Sobre o háhito dos pantópodes se alimentarem de crustáceos, tem-se conhecimento através de observações feitas por RichaRdS \& FRY (1978). Esses autores sugerem que algumas espécies alimentam-se especialmente de anfípodes 
mortos, confirmando-se, assim, os resultados aqui obtidos.

STOCK (1978), testando a preferência de Pantopoda por duas espécies de macroalgas feofícias, características do Hemisfério Norte (Ascophyllum Stackouse, 1809 e Fucus Linnaeus, 1753 ), também verificou que, como neste estudo, foram rejeitadas. BAMBER \& DAVIS (1982) demonstraram, experimentalmente, que pantópodes da espécie Achelia echinata Hodge, 1864 alimentam-se de Enteromorpha Link, 1820 e Griffithsia J. Agardh, 1851.

Quanto à influência do aparelho bucal (probóscide, palpos e quelíforos) no modo de alimentação e no tipo de alimento preferido pelos pantópodes pouco pode-se concluir. De maneira geral, pode-se dizer que, embora a literatura cite os principais alimentos comidos pelos pantópodes eles possuem diferentes graus de preferência assim como formas distintas para sua ingestão pois as espécies apresentam diferentes tipos de probóscides e presença ou ausência de palpos e quelíforos. Nas espécies aqui estudas acredita-se que, as diferenças das peças bucais (Tanystylum isabellae possui palpos e probóscide afilada e Anoplodactylus stictus apresenta quelíforos e probóscide arredondada) não tenham influenciado na escolha do alimento já que ambas espécies comeram os mesmos tipos.

Constatou-se, ainda, que as duas espécies são caracteristicamente carnívoras mostrando preferências por organismos sésseis ou por partes de indivíduos vágeis mortos, o que se atribui à facilidade de inserção da probóscide no alimento para a sua possível sucção e ao pequeno poder de locomoção dos pantópodes.

AGRADECIMENTOS. Este trabalho é parte da minha Dissertação de Mestrado. Sou grata à Profa. Dra, Patricia Teresa Monteiro Cunningham pela revisão e sugestões.

\section{REFERÊNCIAS BIBLIOGRÁFICAS}

ANDRÉ, M. \& E. LAMY. 1938. Pycnogonides parasites de mollusques. J. Conch., Paris, 82: 326-331.

ARnaud, F. 1978. A new species of Ascorhynchus (Pycnogonida) found parasitic on a opistobranchiate mollusc. Zool. J. Linn. Soc. 63 (1/2): 99-105.

Bamber, R.N. \& M.H. Davis. 1982. Feeding of Achelia echinata Hodge (Pycnognida) on marine algae. J. Exp. Mar. Biol Ecol. 60: 181-187.

ChILD, C.A. \& G.R. HARBISON. 1986. A parasitic association between a pycnogonid and a scyphomedusa in midwater. J. mar. biol. Ass. U.K. 66: 113-117.

DE HaRO, A. 1967. Relaciones entre picnogónidos e hidroideos en el medio posidonícola. Boln R. Sox. esp. Hist. nat. 65: 301-303.

FRY, W.G. 1965. The feeding mechanisms and preferred foods of three species of Pycnogonida. Bull. Br. Mus. nat. Hist. Zool. 12 (6): 197-223.

OHshima, H. 1927. Nymphonella tapetis n.g., n.sp. A pycnogon parasitic a bivalve. Annotnes Zool. Japan. 11 (3): 257-263.

1933a. The adult of the bivalve-infesting pycnogonid, Nymphonella tapetis. Annotnes Zool. Japan. 14 (1): 53-60.

1933b. Young pycnogonids found parasitic nudibranchs. Annotnes 
Zool. Japan. 14 (1): 61-66.

Richards, P.R. \& W.G. FRY. 1978. Digestion in pycnogonids: a study of some polar forms. Zool. J. Linn. Soc. 63 (1/2): 75-97.

Sloan, N.A. 1979. Pycnogonid-ophiuroid association. Mar. Biol. 52: 171-176. STOCK, J.H. 1978. Experiments on the food preference and chemical sense in Pycnogonida. Zool. J. Linn. Soc. 63 (1/2): 59-74.

VAROLI, F.M.F. 1981. Aspectos da distribuição, reprodução e alimentação de Pantopoda de Itanhaém e Ubatuba, Estado de São Paulo. Dissertação de Mestrado, não publicada, Instituto Oceanográfico, Universidade de São Paulo, São Paulo, 89p.

Recebido em 15.X.1993; aceito em 08.VIII. 1994. 